\title{
A High-Speed Single Crystal Silicon AFM Probe Integrated with PZT Actuator for High-Speed Imaging Applications
}

\author{
Il-Joo Cho ${ }^{\dagger}$, Kwang Seok Yun* and Hyo-Jin Nam**
}

\begin{abstract}
A new high speed AFM probe has been proposed and fabricated. The probe is integrated with PZT actuated cantilever realized in bulk silicon wafer using heavily boron doped silicon as an etch stop layer. The cantilever thickness can be accurately controlled by the boron diffusion process. Thick SCS cantilever and integrated PZT actuator make it possible to be operated at high speed for fast imaging. The resonant frequency of the fabricated probe is $92.9 \mathrm{kHz}$ and the maximum deflection is $5.3 \mu \mathrm{m}$ at $3 \mathrm{~V}$. The fabricated probe successfully measured the surface of standard sample in an AFM system at the scan speed of $600 \mu \mathrm{m} / \mathrm{sec}$
\end{abstract}

Keywords: AFM, Probe, PZT, Actuator, Boron, Cantilever, Bio imaging

\section{Introduction}

The AFM (Atomic Force Microscopy) system is not only a powerful tool for observing the surface topology with nanometer resolution but also expands its applications to high density data storage [1] and high speed nano lithography [2]. Recently, AFM system has been widely used for bio-imaging application such as protein and live cells imaging which requires fast imaging capability [3,4]. Therefore, increasing the scan speed of the AFM system has been one of the priorities for these bio-imaging applications.

The scanning speed of an AFM system is determined by the mechanical resonant frequency of the probe. Therefore, it is critical to control the probe thickness, and in the case of a fast resonating structure, it is desirable to have a thick probe body. Typically, the speed of AFM system is limited by the bulk actuator which has low resonant frequencies less than $1 \mathrm{kHz}$. Previously, these AFM probes integrated with piezoelectric actuator have been fabricated using silicon or dielectric film. In the case of silicon probes, the probe structure has been fabricated from the device layer of a SOI wafer. However, the final probe thickness cannot be accurately controlled because of the inherent nonuniformity in the SOI device layer thickness as well as the non-uniformity in the silicon dry etching step that defines the probe tip. In case of AFM probes made from dielectric film, the probe thickness is usually limited below $2 \mu \mathrm{m}$ even though low stress silicon nitride film is used. Therefore, it is difficult to achieve high resonant frequency with a dielectric film probe.

\footnotetext{
$\dagger \quad$ Corresponding Author: Nano-Bio Center, Korea Institute of Science and Technology, Korea. (ijcho@kist.re.kr)

* Department of Electronic Engineering, Sogang University, Korea. (ksyun@sogang.ac.kr)

** Devices and Materials Lab, LG Electronics Institute of Technology, Korea. (hyojnam@lge.com)
}

Received: July 20, 2010; Accepted: August 10, 2010
To solve these problems, we report a new AFM probe with integrated PZT (lead zirconate titanate) actuator on the boron doped silicon cantilever as shown in fig. 1. By using boron doped layer as an etch stop in wet releasing process, the probe thickness can be exactly controlled and the thickness can be increased beyond $10 \mu \mathrm{m}$ in order to achieve a higher resonant frequency probe. In addition, the integrated PZT actuator makes it possible to be operated at higher frequency for high speed applications.

The proposed AFM probe exhibits a thick cantilever appropriate for high speed applications and uniform thickness that can be used in the array system, which requires uniformity in its cantilever arrays.

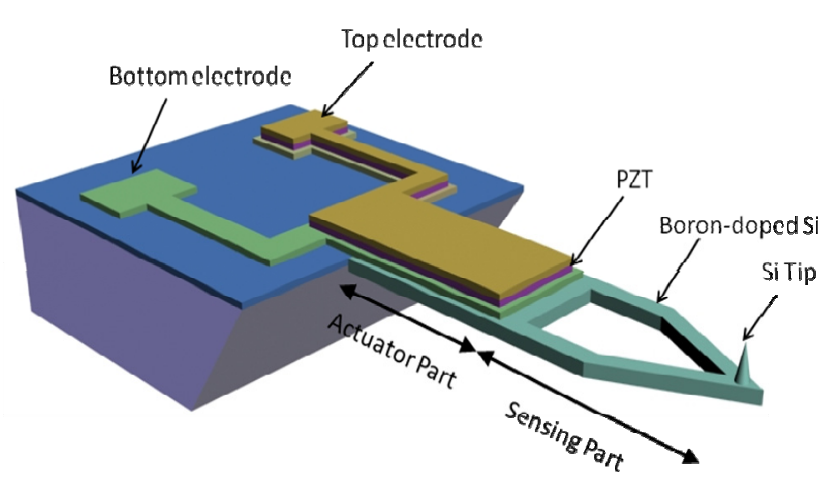

Fig. 1. Proposed AFM probe integrated with PZT actuator.

\section{Structure and Fabrication}

The proposed structure of the high speed AFM probe is shown in fig 1 . The cantilever consists of the actuator part and the force sensing part. To minimize coupling between the actuator part and the force sensing part, the spring constant of the sensing part has been designed to be about 10 times smaller than that of the actuating part. Thus, 
the cantilever deflection induced by the atomic force will be localized in the sensing part of the cantilever. A PZT actuator is integrated to actuate the AFM probe which allows the sharp tip at the sensing end of the probe to scan surfaces with high resolution. Finally, bonding pads are placed on the probe body for electrical connections to apply voltage.

The fabrication process of the proposed AFM probe tip is shown in fig. 2. The starting material is $<100>$ silicon wafer and $1 \mu \mathrm{m}$ thermal oxide is grown. With this oxide as a mask, probe tip is formed by dry etch. Next, another $1 \mu \mathrm{m}$ thermal oxide is grown and patterned as a boron diffusion mask. Then, heavily boron-doped layer is made via boron diffusion from the solid source. By adjusting diffusion time and temperature, cantilever thickness can be easily and accurately controlled [7]. On top of the boron diffusion layer, another oxide layer is grown for insulation. Then, $\mathrm{Pt}$ layer $(150 \mathrm{~nm})$ is sputtered for bottom electrode. The PZT layer with thickness of $280 \mathrm{~nm}$ is formed by sol-gel process. On top of the PZT film, $\mathrm{RuO}_{2}$ is deposited as a top electrode. Then, the PZT actuator structure is patterned by dry etching and $\mathrm{Cr} / \mathrm{Au}$ pads are formed by e-beam evaporation and lift-off processes. By using boron diffusion layers as an etch stop, silicon is etched and the probe is released in TMAH etchant followed by the definition of cantilever region at the front side by $\mathrm{RIE}$ using $\mathrm{Cr}$ as an etch mask. Finally, $\mathrm{Cr}$ is deposited on the backside of the cantilever to enhance the reflectivity of the surface during measurements because an AFM system measures the deflection of the probe by the reflected laser on the backside.

Fig. 3 shows the SEM image of the fabricated probe and the enlarged picture of the PZT actuator. These pictures show that stress-free heavily boron-doped cantilever has been successfully fabricated. The length of the cantilever is $400 \mu \mathrm{m}$ and its thickness is $3.5 \mu \mathrm{m}$. The cantilever thick- (a)

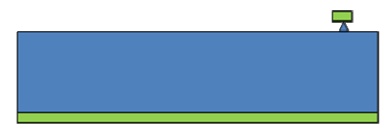

(b)

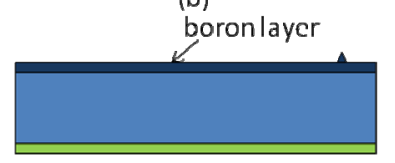

(c)

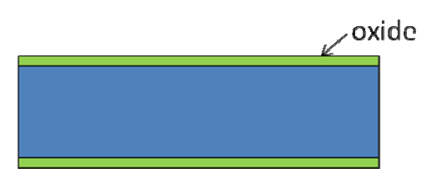

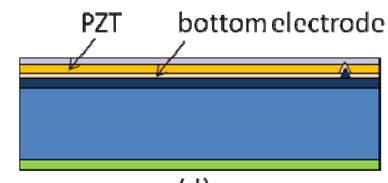

(d)

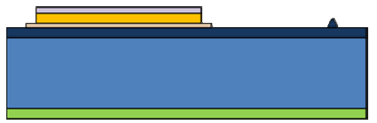

(c)

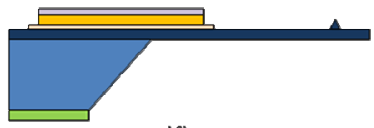

(f)
Fig. 2. Process flow of the proposed AFM probe. (a) oxidation for etching mask, (b) dry etching for tip formation, (c) deep boron diffusion for cantilever structure, (d) bottom electrode/PZT/top electrode formation, (e) electrodes patterning and PZT etching, (f) release in TMAH.

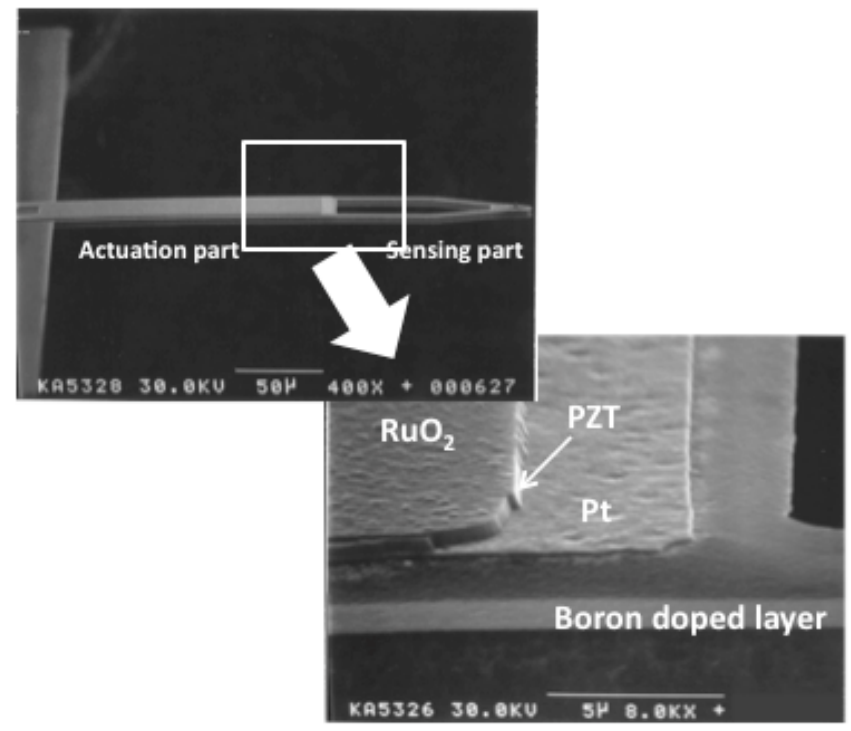

Fig. 3. SEM pictures of the fabricated AFM probe.

ness can be adjusted by controlling boron diffusion time and temperature.

\section{Measurement Results}

\subsection{Mechanical Characteristics}

The mechanical characteristics of the fabricated AFM probe tips have been measured. A sinusoidal wave with $100 \mathrm{mV}$ amplitude was applied to the PZT actuator; the vibration amplitude was monitored by a lock-in amplifier. In response to the voltage actuation, the probe tip showed a resonant frequency and quality factor of $92.9 \mathrm{kHz}$ and 180 , respectively, as shown in Fig. 4 (a). The resonant frequency of the actuating cantilever is more than 100 times higher than the conventional PZT tube scanner and it can be increased by adjusting the cantilever thickness. Fig. 4 (b) shows the measured deflection of the probe tip as a function of actuation voltage. The deflection per volt has been measures to be about $1.8 \mu \mathrm{m} / \mathrm{V}$ and the maximum deflection is $5.3 \mu \mathrm{m}$ at $3 \mathrm{~V}$. A mild hysteresis and non-linear behavior are shown for large displacement. The breakdown voltage of the PZT actuator is $25 \mathrm{~V}$ and the maximum operational range of the AFM system can be $45 \mu \mathrm{m}$ which is much higher than that of the conventional atomic force microscopy.

\subsection{Imaging}

To scan image using the fabricated probe, the AFM measurement system has been modified as an active high speed measurement system. The PZT cantilever is used for controlling the position of the probe tip in the $\mathrm{Z}$ direction. The optical lever method is used to detect the cantilever deflection caused by atomic force acting on the tip. As the probe scans the surface, the tip follows the topology 


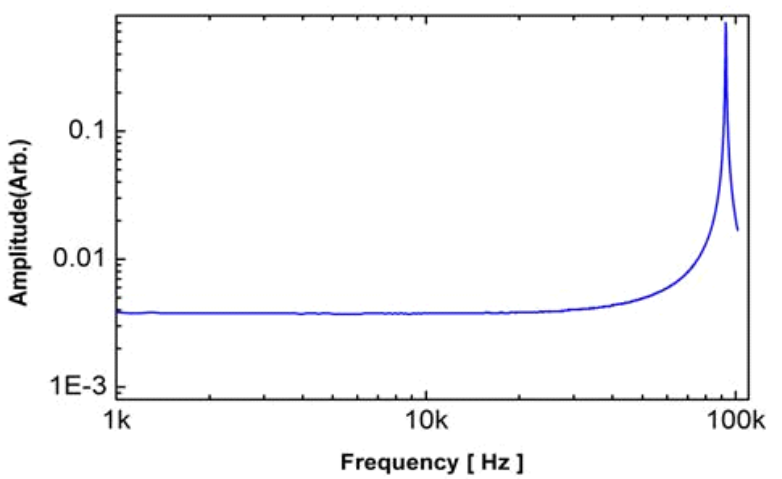

(a)

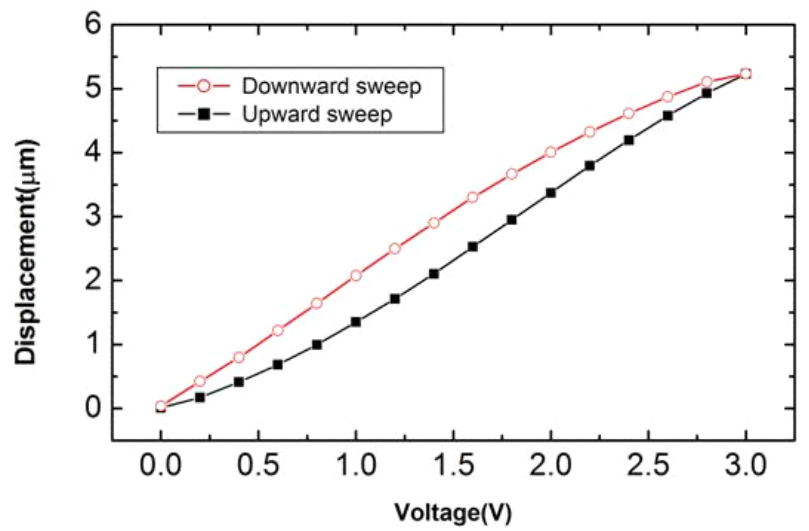

(b)

Fig. 4. Mechanical properties of the fabricated PZT cantilever: (a) frequency response, (b) tip displacement according to the applied voltage.

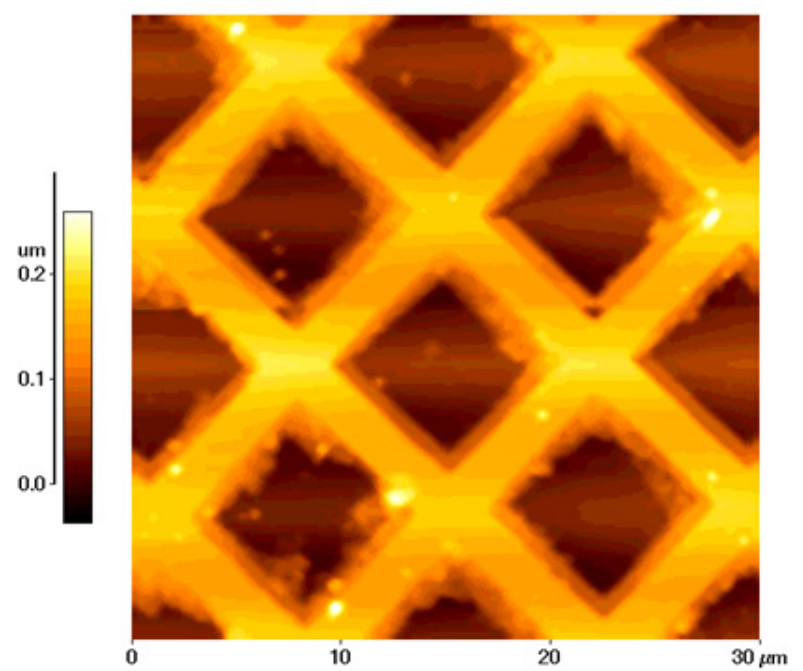

Fig. 5. AFM image scanned by the fabricated PZT integrated AFM probe tip.

changes in the $\mathrm{Z}$ direction. Necessarily, before any scanning can continue, the probe tip has to revert to a certain distance above the surface after the Van der Waals force brings it towards the surface. In conventional AFM systems, external PZT tube scanner is used to maintain the distance between sample surface and probe tip by z-axis actuation in response to a position-sensitive detector. However, the scan speed is limited by the slow external PZT tube scanner because the probe tip cannot be deflected away from the surface fast enough. In this case, the scan speed is about $60 \mu \mathrm{m} / \mathrm{sec}$. In our design, the integrated PZT actuator allows much faster returning speed of the probe tip. Consequently, the scan speed exhibits movements that are nearly 10 times faster than in conventional AFM systems.

Fig. 5 shows AFM images of the standard calibration sample with $10 \mu \mathrm{m}$ period and $100 \mathrm{~nm}$ height. The selfactuating PZT probe is used for positioning the tip in the $\mathrm{z}$ direction. In this experiment, the scan speed is $600 \mu \mathrm{m} / \mathrm{sec}$, The scanned image shows a good image without distortion at high speed.

\section{Conclusions}

We have proposed and fabricated a high speed AFM probe with integrated PZT actuator using a heavily borondoped layer realized in $\langle 100\rangle$ bulk silicon wafer. The cantilever thickness can be easily controlled by adjusting the boron diffusion temperature and diffusion time. Also, this process is CMOS compatible with boron diffusion layer used as an etch stop layer in TMAH. The fabricated PZT actuator has demonstrated its highest tip deflection of $1.8 \mu \mathrm{m} / \mathrm{V}$ at the resonant frequency of $92.9 \mathrm{kHz}$. Also, it can be increased by simply adjusting boron diffusion time and thickness. With the fabricated AFM probe, high-speed feedback actuation can then be carried out, which is beyond the abilities of conventional external bulk PZT tube actuations. High-speed AFM images have been successfully obtained at the scan speed of $600 \mu \mathrm{m} / \mathrm{sec}$. This fabricated high-speed AFM has advantages in bio imaging applications such as proteins and live cells imaging, which require fast imaging capabilities. Moreover, uniformly and accurately controlled probe thickness makes it easier to be used in an array system that requires minimal variation across individual components.

\section{References}

[1] B. Chui, T. Stowe, Y. Ju, K. Goodson, T. Kenny, H. Mamin, B. Terris, R. Ried, and D. Rugar, "Lowstiffness silicon cantilevers with integrated heaters and piezoresistive sensors for high-density AFM thermomechanical data storage", Journal of Microelectromechanical Systems, Vol. 7, p. 69, 1998.

[2] C. Mart n, G. Rius, X. Borrise, and F. Perez-Murano, "Nanolithography on thin layers of PMMA using atomic force microscopy", Nanotechnology, Vol. 16, pp. 1016-1022, 2005.

[3] S. Sen, S. Subramanian and D. E. Discher, "Indentation and adhesive probing of a cell membrane with AFM: theoretical model and experiments", Biophysi- 
cal Journal, Vol. 89, issue 5, pp. 3203-3213, 2005.

[4] D. Fotiadis, S. Scheuring, S. A. Muller, A. Engel and D. J. Muller, "Imaging and manipulation of biological structures with the AFM", Micron, Vol. 33, issue 4, pp. 385-397, 2005.

[5] Y. Kim, H. Nam, S. Cho, J. Hong, D. Kim, and J. Bu, "PZT cantilever array integrated with piezoresistor sensor for high speed parallel operation of AFM," Sensors \& Actuators: A. Physical, Vol. 103, pp. 122129, 2003.

[6] T. Sulchek, R. Hsieh, J. Adams, G. Yaralioglu, S. Minne, C. Quate, J. Cleveland, A. Atalar, and D. Adderton, "High-speed tapping mode imaging with active Q control for atomic force microscopy," Applied Physics Letters, Vol. 76, p. 1473, 2000.

[7] I. Cho, E. Park, S. Hong, and E. Yoon, "Atomic force microscope probe tips using heavily boron-doped silicon cantilevers realized in a $<110>$ bulk silicon wafer," Jpn. J. Appl. Phys, Vol. 39, pp. 7103-7107, 2000.

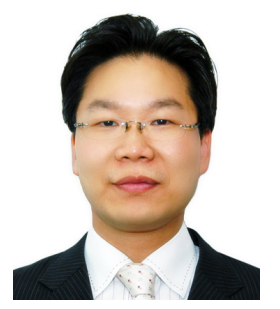

Il-Joo Cho received his B.S, M.S. and $\mathrm{Ph} . \mathrm{D}$ degrees in electrical engineering from the Korean Advanced Institute of Science and Technology (KAIST), Deajeon, Korea, in 1998, 2000, 2004, respectively. From 2004 to 2007 he worked for LG Electronics Institute of Technology. He was a research fellow at the University of Minnesota, Minneapolis from 2007 to 2008. He was a visiting research scientist at the University of Michigan, Ann Arbor from 2008 to 2010. In 2010, he joined Nano-Bio Center at KIST(Korea Institute of Science and Technology). His research interests are in MEMS, micro medical devices, and integrated microsystems.

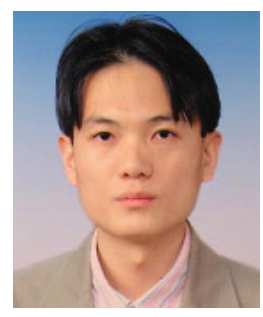

Kwang-Seok Yun received his B.S. degree in Electronics Engineering from Kyungpook National University in 1996, M.S. and Ph.D. degrees in Electronics Engineering from Korea Advanced Institute of Science and Technology (KAIST) in 1999 and 2003, respectively. He was a post-doctorial researcher at University of California, Los Angeles from 2005 to 2007. He joined the Department of electronic Engineering at Sogang University, Korea in 2007, where he is now an Assistant Professor. His current research area includes micro total analysis systems, Labon-a-chip, MEMS, and micro sensors and actuators.

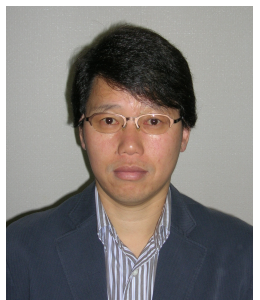

Hyo-Jin Nam received M.S., and Ph. D. degrees in material science from Korea Advanced Institute of Science and Technology (KAIST) in 1988 and 1998, respectively. He joined LG Electronics Institute of Technology in 1988, where he has worked on silicon solar cell, FeRAM and microsensors. His research interests include micro sensors, optical MEMS, RF MEMS, NEMS and probe-based data storage. 